\title{
MODULI OF WEIGHTED POINTED STABLE CURVES AND LOG CANONICAL MODELS OF $\overline{\mathcal{M}}_{g, n}$
}

\author{
MAKSYM FEDORCHUK
}

\begin{abstract}
We establish nefness of certain tautological divisor classes on $\overline{\mathcal{M}}_{0, n}$, and more generally on Hassett's spaces $\overline{\mathcal{M}}_{g, \mathcal{A}}$, and prove that $\overline{\mathcal{M}}_{g, \mathcal{A}}$ is a log canonical model of $\overline{\mathcal{M}}_{g, n}$ for every genus $g$ and every weight vector $\mathcal{A}$.
\end{abstract}

\section{Introduction}

Pointed stable curves are of fundamental importance in algebraic geometry and have found application in such areas as moduli theory, Gromov-Witten theory, resolution of singularities [5], and many others. However, some aspects of birational geometry of Deligne-Mumford-Knudsen spaces $\overline{\mathcal{M}}_{g, n}$ of $n$-pointed stable curves of genus $g$ remain unknown. A fundamental, and still unsolved, problem is to describe the cone of nef divisors on $\overline{\mathcal{M}}_{g, n}$. There is a conjectural description, called the F-conjecture (usually attributed both to Faber and Fulton), of $\operatorname{Nef}\left(\overline{\mathcal{M}}_{g, n}\right)$ that has been established in a number of cases $[6,9,10,15,18]$. In this note we study a collection of naturally defined nef divisor classes on cousins of $\overline{\mathcal{M}}_{g, n}$, the Hassett's spaces $\overline{\mathcal{M}}_{g, \mathcal{A}}$ of weighted pointed stable curves, with a goal of understanding $\operatorname{Nef}\left(\overline{\mathcal{M}}_{0, n}\right)$ and whether $\overline{\mathcal{M}}_{0, n}$ is a Mori dream space [13].

We establish nefness (and in some cases ampleness) of certain divisors on $\overline{\mathcal{M}}_{g, \mathcal{A}}$ by exploiting the polarizing line bundle on the universal family over $\overline{\mathcal{M}}_{g, \mathcal{A}}$, which is known to be nef by Kollár's semipositivity results [17]. As an application, we prove that the coarse moduli space of $\overline{\mathcal{M}}_{g, \mathcal{A}}$ is a log canonical model of $\overline{\mathcal{M}}_{g, n}$ (Theorem 4.1) for every genus $g$ and weight vector $\mathcal{A}$. This solves Problem 7.1 of [12] (see also "Concluding Remarks" of [1]) and generalizes results of [1, 7, 19] to arbitrary genus and non-symmetric weight vector.

Before describing our strategy for producing nef (and ample) divisors on $\overline{\mathcal{M}}_{g, \mathcal{A}}$, we recall the definition of $\overline{\mathcal{M}}_{g, \mathcal{A}}$ from [12]: Given a weight vector $\mathcal{A}=\left(a_{1}, \ldots, a_{n}\right) \in$ $(0,1]^{n} \cap \mathbb{Q}^{n}$, a connected, $n$-pointed, proper curve $\left(C ; p_{1}, \ldots, p_{n}\right)$ of genus $g$ is $\mathcal{A}$-stable if

(1) $C$ has at worst nodal $(x y=0)$ singularities, and $p_{1}, \ldots, p_{n}$ are smooth points of $C$.

(2) $\omega_{C}\left(\sum_{i=1}^{n} a_{i} p_{i}\right)$ is ample.

(3) $\operatorname{mult}_{x} \sum_{i=1}^{n} a_{i} p_{i} \leq 1, \forall x \in C$.

$\overline{\mathcal{M}}_{g, \mathcal{A}}$ is the moduli stack whose objects are flat families with $\mathcal{A}$-stable geometric fibers of genus $g$.

Received by the editors November 4, 2010. Revision received April 19, 2011. 
Now consider the universal family $\left(\pi: \mathcal{C} \rightarrow \overline{\mathcal{M}}_{g, \mathcal{A}} ;\left\{\sigma_{i}\right\}_{i=1}^{n}\right)$ of $\mathcal{A}$-stable curves. From the definition above, the line bundle $\omega_{\pi}\left(\sum_{i=1}^{n} a_{i} \sigma_{i}\right)$ is a priori only $\pi$-nef. However $\omega_{\pi}\left(\sum_{i=1}^{n} a_{i} \sigma_{i}\right)$ turns out to be nef on $\mathcal{C}$ and its positivity is a key tool in our construction of nef tautological divisors on $\overline{\mathcal{M}}_{g, \mathcal{A}}$. Indeed, the nef divisors that we produce are linear effective combinations of $\pi_{*}\left(\omega_{\pi}\left(\sum_{i=1}^{n} a_{i} \sigma_{i}\right)^{2}\right)$ and $\pi_{*}\left(\omega_{\pi}\left(\sum_{i=1}^{n} a_{i} \sigma_{i}\right) \cdot \sigma_{i}\right)$.

That $\omega_{\pi}\left(\sum_{i=1}^{n} a_{i} \sigma_{i}\right)$ is nef on $\mathcal{C}$ was proved by Kollár in [17, Corollary 4.6 and Proposition 4.7]. When $a_{1}=\cdots=a_{n}=1$, i.e., when $\overline{\mathcal{M}}_{g, \mathcal{A}}=\overline{\mathcal{M}}_{g, n}$, the nefness of $\omega_{\pi}\left(\sum_{i=1}^{n} \sigma_{i}\right)$ was also established by Keel [14, Theorem 0.4]. We give yet another proof that $\omega_{\pi}\left(\sum_{i=1}^{n} a_{i} \sigma_{i}\right)$ is nef in Proposition 2.1; the argument is elementary and is included because it gives a finer information of curves on which $\omega_{\pi}\left(\sum_{i=1}^{n} a_{i} \sigma_{i}\right)$ has degree 0 .

Even though we phrase all the results for an arbitrary genus, the positive genus case of Theorem 4.1 follows essentially from that of genus 0 via a well-known positivity result on $\overline{\mathcal{M}}_{g}$. For this reason, we isolate nef tautological divisors on $\overline{\mathcal{M}}_{0, n}$ produced by our construction in Section 5 . These include all divisors contained in the GIT cone of $\overline{\mathcal{M}}_{0, n}$ [1], as we prove in Proposition 5.1. We leave open the question whether two cones are in fact the same.

We work over an algebraically closed field of arbitrary characteristic.

\section{Main positivity result}

Situation 1. Let $B$ be a smooth complete curve. Consider a flat proper family $\pi: \mathcal{C} \rightarrow B$ of connected at worst nodal curves of arithmetic genus $g$. Suppose that $\pi$ has $n$, not necessarily distinct, sections

$$
\sigma_{1}, \ldots, \sigma_{n}: B \rightarrow \mathcal{C}
$$

such that every $\Sigma_{i}:=\sigma_{i}(B)$ lies in $\mathcal{C} \backslash \operatorname{Sing}(\mathcal{C} / B)$. We denote both the relative dualizing line bundle of $\pi$ and its numerical class by $\omega$. We denote the numerical class of a fiber by $F$. In what follows, we repeatedly use the adjunction formula $\omega \cdot \Sigma_{i}+\Sigma_{i}^{2}=0$. An effective divisor $C \subset \mathcal{C}$ is called vertical if $\pi(C)$ is a point.

Proposition 2.1. Let $\pi: \mathcal{C} \rightarrow B$ be a generically smooth family of curves as in Situation 1. Suppose that for a weight vector $\mathcal{A}=\left(a_{1}, \ldots, a_{n}\right) \subset(0,1]^{n} \cap \mathbb{Q}^{n}$ the line bundle

$$
L:=\omega+\sum_{i=1}^{n} a_{i} \Sigma_{i}
$$

is $\pi$-nef. Suppose further that $\Sigma_{i_{1}}=\cdots=\Sigma_{i_{k}}$ (for some $i_{1}<\cdots<i_{k}$ ) only if $\sum_{\ell=1}^{k} a_{i_{\ell}} \leq 1$. Then $L$ is a nef divisor on $\mathcal{C}$.

Furthermore, if $L$ is $\pi$-ample, then $L$ has positive degree on all curves $C \subset \mathcal{C}$ with the following exceptions:

(1) $C=\Sigma_{i_{1}}=\cdots=\Sigma_{i_{k}}$ for some $i_{1}<\cdots<i_{k}$ satisfying $\sum_{\ell=1}^{k} a_{i_{\ell}}=1$.

(2) $\left(\pi: \mathcal{C} \rightarrow B ;\left\{\sigma_{i}\right\}_{i=1}^{n}\right)$ is a constant family and $C$ is a constant section.

Remark 1. The assumptions of the above proposition are weaker than the condition of $\mathcal{A}$-stability (see Section 1) in that they allow a collection of sections of total weight greater than 1 to intersect at finitely many points. 
Proof. The surface $\mathcal{C}$ has at worst $A_{k}$ singularities, which are Du Val (the discrepancies of the canonical divisor are zero). It follows that the statement holds for $\mathcal{C}$ if and only if it holds for the minimal desingularization of $\mathcal{C}$. From now on, we assume that $\mathcal{C}$ is smooth. In what follows, we also repeatedly use the following observation: for any curve $C \subset \mathcal{C}$

$$
\omega \cdot C>0 \Longrightarrow L \cdot C \geq 0
$$

with equality achieved only if $C$ coincides with sections $\Sigma_{i}$ of total weight 1 . Indeed, if $C=\Sigma_{j_{1}}=\cdots=\Sigma_{j_{k}}$ are all the sections coinciding with $C$, then

$$
\begin{aligned}
L \cdot C & \geq\left(\omega+\sum_{\ell=1}^{k} a_{j_{\ell}} \Sigma_{j_{\ell}}\right) \cdot C \\
& =\left(1-\sum_{\ell=1}^{k} a_{j_{\ell}}\right)(\omega \cdot C) .
\end{aligned}
$$

Proof in the case $g \geq 2$. Consider an irreducible curve $C \subset \mathcal{C}$ not contained in any fiber of $\pi$. Denote by $f: \mathcal{C} \rightarrow \mathcal{C}_{\text {min }}$ the relative minimal model of $\mathcal{C}$ over $B$. We have $\omega=f^{*} \omega_{\mathcal{C}_{\min } / B}+E$, where $E$ is an effective vertical divisor. If $\mathcal{C}_{\text {min }} \rightarrow B$ is nonconstant, then consider the relative canonical model $\mathcal{C}_{\text {min }} \rightarrow \mathcal{C}_{\text {can }}$. By $[11$, Theorem $6.33] \omega_{\mathcal{C}_{\text {can }} / B}$ is ample. Hence $\omega_{\mathcal{C}_{\text {min }} / B}$ is nef and has positive degree on every nonvertical curve on $\mathcal{C}_{\text {min }}$. It follows that $\omega \cdot C=\left(f^{*} \omega_{\mathcal{C}_{\min } / B}+E\right) \cdot C>0$. By the observation above, we conclude that $L \cdot C \geq 0$, with equality achieved only when $C$ coincides with sections of total weight 1 . If $\mathcal{C}_{\text {min }} \rightarrow B$ is a constant family, then $\omega \cdot C=\omega_{\mathcal{C}_{\min } / B} \cdot f(C)+E \cdot C \geq 0$, and equality is achieved only when $\Sigma:=f(C)$ is a constant section and $C \cdot E=0$. These conditions can be satisfied only if $(\pi: \mathcal{C} \rightarrow$ $\left.B ;\left\{\sigma_{i}\right\}_{i=1}^{n}\right)$ is a constant family.

Proof in the case $g=1$. Denote by $f: \mathcal{C} \rightarrow \mathcal{C}_{\text {min }}$ the relative minimal model of $\mathcal{C}$ over $B$. We have $\omega=f^{*} \omega_{\mathcal{C}_{\min } / B}+E$, where $E$ is an effective vertical divisor. By Kodaira's formula for the canonical bundle of an elliptic surface $\omega_{\mathcal{C}_{\min } / B}=d F$, where $d \geq 0$, and $d=0$ if and only if $\mathcal{C}_{\text {min }} \rightarrow B$ is a constant family [8, Theorem 15 and Corollary 17, pp. 176-177].

Consider now an irreducible curve $C \subset \mathcal{C}$ not contained in any fiber of $\pi$. If $\mathcal{C}_{\min } \rightarrow B$ is constant, then $\omega \cdot C=E \cdot C \geq 0$ with the equality achieved only if $\left(\pi: \mathcal{C} \rightarrow B ;\left\{\sigma_{i}\right\}_{i=1}^{n}\right)$ is a constant family. Suppose that $\mathcal{C}_{\min } \rightarrow B$ is non-constant, then $\omega \cdot C=d(F \cdot C)+E \cdot C>0$. Again, by the observation above, this implies that $L \cdot C \geq 0$, with equality achieved only when $C$ coincides with sections of total weight 1 .

Proof in the case $g=0$. Consider an irreducible curve $C \subset \mathcal{C}$ not contained in any fiber of $\pi$. The proof breaks into two parts: in the first, we deal with the case when $C$ is a section; in the second, we show that the general case reduces to the former.

First, suppose that $C$ is a section. By successively blowing-down (-1)-curves not meeting $C$, we reduce to the case when $\mathcal{C} \rightarrow B$ is a $\mathbb{P}^{1}$-bundle. 
Case (a): $C$ is a negative section of $\mathcal{C} \rightarrow B$. Without loss of generality, we can assume that sections $\Sigma_{1}, \ldots, \Sigma_{k}$ coincide with $C$. Then compute

$$
\begin{aligned}
L \cdot C & =\left(\omega+\sum_{i=1}^{n} a_{i} \Sigma_{i}\right) \cdot C \\
& =-C^{2}+\sum_{i=1}^{k} a_{i} C^{2}+\sum_{i=k+1}^{n} a_{i}\left(\Sigma_{i} \cdot C\right) \\
& =-C^{2}\left(1-\sum_{i=1}^{k} a_{i}\right)+\sum_{i=k+1}^{n} a_{i}\left(\Sigma_{i} \cdot C\right) \geq 0,
\end{aligned}
$$

where equality can be achieved only when $\sum_{i=1}^{k} a_{i}=1$.

Case (b): $C$ is a non-negative section and $\mathcal{C} \rightarrow B$ has a negative section. To make the computation more transparent, let $\left\{\Sigma_{i}\right\}_{i=1}^{k},\left\{\Sigma_{i}\right\}_{i=k+1}^{\ell}$, and $\left\{\Sigma_{i}\right\}_{i=\ell+1}^{n}$ be the sections that, respectively, coincide with $C$, have negative self-intersection, and are neither of the first two. Let $E$ be the unique section of negative self-intersection, say, $E^{2}=-r<0$. In particular, $\Sigma_{i}=E$ for all $i=k+1, \ldots, \ell$. By assumption, we have

$$
\begin{gathered}
\sum_{i=1}^{k} a_{i} \leq 1, \\
\sum_{i=k+1}^{\ell} a_{i} \leq 1, \\
\sum_{i=1}^{n} a_{i} \geq 2 .
\end{gathered}
$$

Any non-negative section $\Sigma$ on $\mathcal{C}$ satisfies $\Sigma^{2} \geq r$. Also, since $\left(C-\Sigma_{i}\right)^{2}=0$, we have

$$
C \cdot \Sigma_{i}=\frac{1}{2}\left(C^{2}+\Sigma_{i}^{2}\right), \quad i=1, \ldots, n .
$$

Now compute

$$
\begin{aligned}
L \cdot C & =\left(\omega+\sum_{i=1}^{n} a_{i} \Sigma_{i}\right) \cdot C \\
& =-C^{2}+\sum_{i=1}^{n} a_{i}\left(\Sigma_{i} \cdot C\right) \\
& =C^{2}\left(\sum_{i=1}^{k} a_{i}+\sum_{i=k+1}^{n} \frac{a_{i}}{2}-1\right)+\frac{1}{2}\left(-r \sum_{i=k+1}^{\ell} a_{i}+\sum_{i=\ell+1}^{n} a_{i} \Sigma_{i}^{2}\right) \\
& \geq r\left(\sum_{i=1}^{k} a_{i}+\sum_{i=\ell+1}^{n} a_{i}-1\right) \geq 0
\end{aligned}
$$

where equality is achieved only if $\sum_{i=1}^{n} a_{i}=2$ and $\sum_{i=1}^{k} a_{i}=1$. 
Case (c): All sections of $\mathcal{C} \rightarrow B$ have non-negative self-intersection. In this case, we have

$$
\begin{aligned}
L \cdot C & =-C^{2}+\sum_{i=1}^{n} a_{i}\left(\Sigma_{i} \cdot C\right)=-C^{2}+\frac{1}{2} \sum_{i=1}^{n} a_{i}\left(\Sigma_{i}^{2}+C^{2}\right) \\
& =\frac{1}{2}\left(\sum_{i=1}^{n} a_{i}-2\right) C^{2}+\frac{1}{2} \sum_{i=1}^{n} a_{i} \Sigma_{i}^{2} \geq 0,
\end{aligned}
$$

where equality is achieved only if $\left(\pi: \mathcal{C} \rightarrow B ;\left\{\sigma_{i}\right\}_{i=1}^{n}\right)$ is a constant family.

Finally, we consider the general case when $C$ is an arbitrary irreducible curve on $\mathcal{C}$ that does not lie in a fiber of $\pi$. Consider the induced map $C \rightarrow B$ and the fiber product $\mathcal{C}^{\prime}:=\mathcal{C} \times{ }_{B} C$.

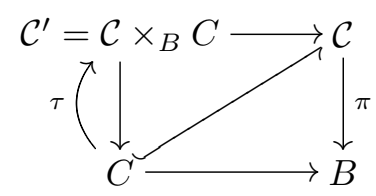

The projection $\mathcal{C}^{\prime} \rightarrow C$ has a section $\tau: C \rightarrow \mathcal{C}^{\prime}$, whose image in $\mathcal{C}^{\prime}$ maps onto $C$ in $\mathcal{C}$. Let $\Sigma_{i}^{\prime}$ be the preimage of $\Sigma_{i}$ on $\mathcal{C}$. Introduce the line bundle

$$
L^{\prime}:=\omega_{\mathcal{C}^{\prime} / C}+\sum_{i=1}^{n} a_{i} \Sigma_{i}^{\prime} .
$$

Then $L^{\prime}$ is the pullback of $L$ from $\mathcal{C}$. Hence $L^{\prime} \cdot \tau(C)=L \cdot C$ by the projection formula. Noting that the surface $\mathcal{C}^{\prime}$ and the line bundle $L^{\prime}$ satisfy the assumptions of the proposition, we have reduced to the case when $\tau(C)$ is a section.

\section{Nef tautological divisors on $\overline{\mathcal{M}}_{g, \mathcal{A}}$}

3.1. Divisor classes on $\overline{\mathcal{M}}_{g, \mathcal{A}}$. We apply the positivity result of Proposition 2.1 to produce nef divisors on the moduli space $\overline{\mathcal{M}}_{g, \mathcal{A}}$ parameterizing $n$-pointed $\mathcal{A}$-stable curves of arithmetic genus $g$. We begin by introducing notation for the divisor classes that we encounter. Recall that $\overline{\mathcal{M}}_{g, \mathcal{A}}$ is a smooth Deligne-Mumford stack, with a projective coarse moduli space $\bar{M}_{g, \mathcal{A}}$, and hence carries a universal family, which we denote $\left(\pi: \mathcal{C} \rightarrow \overline{\mathcal{M}}_{g, \mathcal{A}} ; \sigma_{1}, \ldots, \sigma_{n}\right)[12]$. The following tautological divisor classes are defined on the stack $\overline{\mathcal{M}}_{g, \mathcal{A}}$ :

(1) The Hodge class $\lambda=c_{1}\left(\pi_{*} \omega_{\pi}\right)$.

(2) The kappa class $\kappa=\pi_{*}\left(c_{1}^{2}\left(\omega_{\pi}\right)\right)$ (this is different from $\kappa_{1}$ of [3]).

(3) The psi-classes $\psi_{i}=\pi_{*}\left(-\sigma_{i}^{2}\right)=c_{1}\left(\sigma_{i}^{*}\left(\omega_{\pi}\right)\right)$; the total psi-class is $\psi:=$ $\sum_{i=1}^{n} \psi_{i}$.

(4) The boundary divisors $\Delta_{i, j}:=\pi_{*}\left(\sigma_{i} \cdot \sigma_{j}\right)$. (These are denoted $D_{I, J}(\mathcal{A})$, where $I=\{i, j\}$ and $J=\{1, \ldots, n\} \backslash I$, in [12].) We denote the sum of all $\Delta_{i, j}$ by $\Delta_{s}$.

(5) The sum, denoted $\Delta_{\text {nodal, }}$, of all boundary divisors parameterizing nodal curves. (This divisor class is denoted by $\nu$ in [12].)

Mumford's relation gives $\kappa=12 \lambda-\Delta_{\text {nodal }}$ (cf. [3]). 
In the case of $\overline{\mathcal{M}}_{g, n}$, we also consider boundary divisors $\Delta_{S}$, for every subset $S \subset\{1, \ldots, n\}$ satisfying $|S| \geq 2$ (and in the case of $g=0, n-|S| \geq 2$ ). The generic point of $\Delta_{S}$ is a reducible curve with a single node and an irreducible component of genus 0 marked by sections $\left\{\sigma_{i}\right\}_{i \in S}$. We let $\Delta_{r}$ be the union of all boundary divisors $\Delta_{S}$ with $|S|=r$. Finally, we use the usual notation $\Delta$ for the total boundary of $\overline{\mathcal{M}}_{g, n}$. Note that under the natural reduction morphism $\overline{\mathcal{M}}_{g, n} \rightarrow \overline{\mathcal{M}}_{g, \mathcal{A}}$, the divisor $\Delta$ in $\overline{\mathcal{M}}_{g, n}$ pushes forward to $\Delta_{s}+\Delta_{\text {nodal }}$.

\subsection{Main theorem.}

Theorem 3.1. Let $\mathcal{A}=\left(a_{1}, \ldots, a_{n}\right)$ be a weight vector with $a_{i} \in(0,1] \cap \mathbb{Q}$ and $g$ be a non-negative integer satisfying $2 g-2+\sum_{i=1}^{n} a_{i}>2$. Then the following divisors:

$$
\begin{aligned}
A=A\left(a_{1}, \ldots, a_{n}\right) & :=\kappa+\psi+\sum_{i<j}\left(a_{i}+a_{j}\right) \Delta_{i j} \\
B=B\left(a_{1}, \ldots, a_{n}\right) & :=\kappa+\sum_{i=1}^{n}\left(2 a_{i}-a_{i}^{2}\right) \psi_{i}+\sum_{i<j}\left(2 a_{i} a_{j}\right) \Delta_{i j} \\
C_{i}=C_{i}\left(a_{1}, \ldots, a_{n}\right) & :=\left(1-a_{i}\right) \psi_{i}+\sum_{j \neq i} a_{j} \Delta_{i j}, \quad \text { for each } i=1, \ldots, n,
\end{aligned}
$$

are nef on $\overline{\mathcal{M}}_{g, \mathcal{A}}$.

Proof. We adopt the convention that for a generically singular family $\mathcal{C} \rightarrow T$, sections $T \rightarrow \mathcal{C}$ associated to the conductor have weight 1 . Then the divisors under consideration are functorial with respect to the boundary stratification ${ }^{1}$. Therefore, it suffices to check that they have non-negative degree on every curve $\left(\mathcal{C} \rightarrow T ; \sigma_{1}, \ldots, \sigma_{n}\right)$ with a normal total space $\mathcal{C}$.

To begin, by Proposition 2.1 the divisor

$$
L:=\omega_{\mathcal{C} / T}+\sum_{i=1}^{n} a_{i} \sigma_{i}
$$

is nef on $\mathcal{C}$. In particular, it is pseudoeffective and has non-negative self-intersection. We will show that the intersection numbers $A \cdot T, B \cdot T$, and $C_{i} \cdot T$ are non-negative by expressing each of them as an intersection of $L$ with a pseudoeffective class on $\mathcal{C}$.

For $A$, we note that $\omega_{\mathcal{C} / T}+\sum_{i=1}^{n} \sigma_{i}$ is an effective combination of $L$ and $\left\{\sigma_{i}\right\}_{i=1}^{n}$. Therefore,

$$
0 \leq\left(\omega_{\mathcal{C} / T}+\sum_{i=1}^{n} \sigma_{i}\right) \cdot L=\kappa+\psi+\sum_{i<j}\left(a_{i}+a_{j}\right) \Delta_{i j}=A \cdot T
$$

\footnotetext{
${ }^{1} \mathrm{~A}$ divisor $D$ is functorial with respect to the boundary stratification if for any family $\mathcal{C} \rightarrow T$ whose generic fiber has a node separating connected components of genus $g_{1}$ and $g_{2}$, marked by sections of weights, respectively, $\left(a_{1}, \ldots, a_{k}\right)$ and $\left(a_{k+1}, \ldots, a_{n}\right)$, we have

$$
(D \cdot T)_{\overline{\mathcal{M}}_{g, \mathcal{A}}}=(D \cdot T)_{\overline{\mathcal{M}}_{g_{1},\left(1, a_{1}, \ldots, a_{k}\right)}}+(D \cdot T)_{\overline{\mathcal{M}}_{g_{2},\left(1, a_{k+1}, \ldots, a_{n}\right)}},
$$

where divisors on the right come from families of curves in $\overline{\mathcal{M}}_{g_{1},\left(1, a_{1}, \ldots, a_{k}\right)}$ and $\overline{\mathcal{M}}_{g_{2},\left(1, a_{k+1}, \ldots, a_{n}\right)}$ obtained by normalizing $\mathcal{C}$ along the generic node (cf. [7, Section 2.1]).
} 
For $B$, we have

$$
0 \leq L^{2}=\left(\omega_{\mathcal{C} / T}+\sum_{i=1}^{n} a_{i} \sigma_{i}\right)^{2}=\kappa+\sum_{i=1}^{n}\left(2 a_{i}-a_{i}^{2}\right) \psi_{i}+\sum_{i<j}\left(2 a_{i} a_{j}\right) \Delta_{i j}=B \cdot T .
$$

For $C_{i}$, we have

$$
0 \leq L \cdot \sigma_{i}=\left(1-a_{i}\right) \psi_{i}+\sum_{j \neq i} a_{j} \Delta_{i j}=C_{i} \cdot T .
$$

3.3. Ampleness result. With a bit more work, we can prove that the divisor $A$ is ample. We begin with a few preliminaries.

Definition 1. Let $\mathcal{A}=\left(a_{1}, \ldots, a_{n}\right)$ be a weight vector. Suppose that $a_{1}=\sum_{\ell=1}^{k} b_{\ell}$. Set $\mathcal{B}=\left(b_{1}, \ldots, b_{k}, a_{2}, \ldots, a_{n}\right)$, i.e., $b_{k+i}=a_{i+1}$ for $i \geq 1$.

A replacement morphism $\chi: \overline{\mathcal{M}}_{g, \mathcal{A}} \rightarrow \overline{\mathcal{M}}_{g, \mathcal{B}}$ is a morphism which sends an $\mathcal{A}$-stable curve $\left(\mathcal{C} ;\left\{\sigma_{i}\right\}_{i=1}^{n}\right)$ to a $\mathcal{B}$-stable curve $\left(\mathcal{C} ;\left\{\tau_{j}\right\}_{j=1}^{n+k-1}\right)$, where $\tau_{1}=\cdots=$ $\tau_{k}=\sigma_{1}$ and $\tau_{k+i}=\sigma_{i+1}$ for $i \geq 1$.

Lemma 3.1 (Pull-back formula for $\chi$ ). Keep the notation of Definition 1 and Theorem 3.1. Under the replacement morphism $\chi: \overline{\mathcal{M}}_{g, \mathcal{A}} \rightarrow \overline{\mathcal{M}}_{g, \mathcal{B}}$ the divisor $A\left(b_{1}, \ldots, b_{n+k-1}\right)$ pulls back according to the following formula

$$
\chi^{*}\left(A\left(b_{1}, \ldots, b_{n+k-1}\right)\right)=A\left(a_{1}, \ldots, a_{n}\right)+(k-1) C_{1}\left(a_{1}, \ldots, a_{n}\right) .
$$

Proof. Consider the universal family $\left(\pi: \mathcal{C} \rightarrow \overline{\mathcal{M}}_{g, \mathcal{A}} ;\left\{\sigma_{i}\right\}_{i=1}^{n}\right)$. Then the corresponding family of $\mathcal{B}$-stable curves is $\left(\pi: \mathcal{C} \rightarrow \overline{\mathcal{M}}_{g, \mathcal{A}} ;\left\{\tau_{i}\right\}_{i=1}^{n+k-1}\right)$ where $\tau_{1}=\cdots=\tau_{k}=\sigma_{1}$ and $\tau_{k+i}=\sigma_{i+1}$ for $i \geq 1$. We compute

$$
\begin{aligned}
\chi^{*}\left(A\left(b_{1}, \ldots, b_{n+k-1}\right)\right) & =\pi_{*}\left(\left(\omega_{\pi}+\sum_{i=1}^{n+k-1} \tau_{i}\right)\left(\omega_{\pi}+\sum_{i=1}^{n+k-1} b_{i} \tau_{i}\right)\right) \\
& =\pi_{*}\left(\left(\omega_{\pi}+\sum_{i=1}^{n} \sigma_{i}+(k-1) \tau_{1}\right)\left(\omega_{\pi}+\sum_{i=1}^{n} a_{i} \sigma_{i}\right)\right) \\
& =A\left(a_{1}, \ldots, a_{n}\right)+(k-1) C_{1}\left(a_{1}, \ldots, a_{n}\right) .
\end{aligned}
$$

Lemma 3.2. The divisor $\kappa+\psi$ on $\overline{\mathcal{M}}_{0, \mathcal{A}}$ is a positive linear combination of all irreducible components of $\Delta_{s}$ and $\Delta_{\text {nodal }}$.

Proof. On $\overline{\mathcal{M}}_{0, n}$, we have well-known relations

$$
\psi=\sum_{r=2}^{\lfloor n / 2\rfloor} \frac{r(n-r)}{n-1} \Delta_{r} \quad \text { and } \quad \kappa=-\Delta .
$$

Hence

$$
\kappa+\psi=\sum_{r=2}^{\lfloor n / 2\rfloor} \frac{r(n-r)-n+1}{n-1} \Delta_{r}
$$


For $n \geq 4$, this is a positive linear combination of all irreducible components of $\Delta_{2}+\cdots+\Delta_{\lfloor n / 2\rfloor}$. The general case follows by pushing forward the relation (1) to $\overline{\mathcal{M}}_{0, \mathcal{A}}$ via the reduction morphism.

Proposition 3.1. The divisor $A=A\left(a_{1}, \ldots, a_{n}\right)=\kappa+\psi+\sum_{i<j}\left(a_{i}+a_{j}\right) \Delta_{i j}$ on $\overline{\mathcal{M}}_{g, \mathcal{A}}$ is a pullback of an ample divisor on the coarse moduli space $\bar{M}_{g, \mathcal{A}}$.

Proof. We claim that a sufficiently small neighborhood (in the Euclidean topology with the usual norm $\|\cdot\|)$ of $A$ lies inside the nef cone $\operatorname{Nef}\left(\overline{\mathcal{M}}_{g, \mathcal{A}}\right)_{\mathbb{Q}} \subset \operatorname{NS}\left(\overline{\mathcal{M}}_{g, \mathcal{A}}\right) \otimes \mathbb{Q}$. The statement then follows from Kleiman's criterion [16] applied to the coarse moduli space of $\overline{\mathcal{M}}_{g, \mathcal{A}}$. The proof is by induction on dimension. When dimension is 1 (i.e., when $g=0$ and $|\mathcal{A}|=4$, or $g=1$ and $|\mathcal{A}|=1$ ), the statement is evident.

Suppose $\operatorname{dim} \overline{\mathcal{M}}_{g, \mathcal{A}}>1$. By the functoriality of $A$ and by the induction assumption, a sufficiently small perturbation of $A$ is still ample when restricted to any boundary divisor in $\Delta_{\text {nodal }}$. It remains to show that for any $P \in \mathrm{NS}\left(\overline{\mathcal{M}}_{g, \mathcal{A}}\right) \otimes \mathbb{Q}$ satisfying $\|P\| \ll 1$, the perturbed divisor $A+P$ has non-negative degree on any family $\mathcal{C} \rightarrow B$ with a generically smooth fiber of genus $g$.

The proof falls into two parts: $g=0$ and $g \geq 1$.

Case of $g=0$ : First, suppose that no sections are coincident. Then for $0<\epsilon \ll 1$ the divisor $(1-\epsilon) \omega+\sum a_{i} \sigma_{i}$ is still nef on $\mathcal{C}$ by Proposition 2.1 (see also Remark 1 ). It follows that the divisor

$$
A(\epsilon)=\left((1-\epsilon) \omega+\sum a_{i} \sigma_{i}\right)\left(\omega+\sum \sigma_{i}\right)=A-\epsilon(\kappa+\psi)
$$

is non-negative on $B$. By Lemma 3.2, the divisor $\kappa+\psi$ is a positive linear combination of all irreducible components of $\Delta_{s}$ and $\Delta_{\text {nodal }}$. Since these generate $\operatorname{NS}\left(\overline{\mathcal{M}}_{0, \mathcal{A}}\right)$, we conclude that $\epsilon(\kappa+\psi)+P$ is an effective linear combination of the boundary divisors. Therefore, $A+P=A(\epsilon)+\epsilon(\kappa+\psi)+P$ intersects $B$ non-negatively.

Suppose that $k \geq 2$ sections $\left\{\sigma_{i}\right\}_{i=1}^{k}$ satisfying $\sum_{i=1}^{k} a_{i} \leq 1$ are coincident. Take $\mathcal{A}^{\prime}=\left(\sum_{i=1}^{k} a_{i}, a_{2}, \ldots, a_{n}\right)$ and let $\chi: \overline{\mathcal{M}}_{0, \mathcal{A}^{\prime}} \rightarrow \overline{\mathcal{M}}_{0, \mathcal{A}}$ be the (closed immersion) replacement morphism replacing a section of weight $\sum_{\ell=1}^{k} a_{i_{\ell}}$ by $k$ sections $\left\{\sigma_{i}\right\}_{i=1}^{k}$ of weights $\left\{a_{i}\right\}_{i=1}^{k}$ (see Definition 1 ). Then by Lemma 3.1

$$
\chi^{*}\left(A\left(a_{1}, \ldots, a_{n}\right)\right)=A\left(\sum_{i=1}^{k} a_{i}, a_{2}, \ldots, a_{n}\right)+(k-1) C_{1}\left(\sum_{i=1}^{k} a_{i}, a_{2}, \ldots, a_{n}\right) .
$$

By the induction assumption ${ }^{2}$, the divisor $A\left(\sum_{i=1}^{k} a_{i}, a_{2}, \ldots, a_{n}\right)$ is ample on $\overline{\mathcal{M}}_{0, \mathcal{A}^{\prime}}$. By Theorem 3.1, the divisor $C_{1}\left(\sum_{i=1}^{k} a_{i}, a_{2}, \ldots, a_{n}\right)$ is nef. In particular, the divisor $A+P$ pulls back to the sum of an ample divisor and a nef divisor. It follows from the projection formula that $(A+P) \cdot B \geq 0$.

Case of $g \geq 1$ : Denote by $f: \overline{\mathcal{M}}_{g, n} \rightarrow \overline{\mathcal{M}}_{g, \mathcal{A}}$ the contraction morphism.

Suppose that no sections of $\left(\mathcal{C} \rightarrow B ; \sigma_{1}, \ldots, \sigma_{n}\right)$ are coincident. Then $B$ is not entirely contained in $f(\operatorname{Exc}(f))$. As in the case of $g=0$, for $0<\epsilon \ll 1$, the divisor $(1-\epsilon) \omega+\sum a_{i} \sigma_{i}$ is nef on $\mathcal{C}$. It follows that the divisor

$$
A(\epsilon)=\left((1-\epsilon) \omega+\sum a_{i} \sigma_{i}\right)\left(\omega+\sum \sigma_{i}\right)=A-\epsilon(\kappa+\psi)
$$

\footnotetext{
${ }^{2}$ Note that $\operatorname{dim}\left(\overline{\mathcal{M}}_{0, \mathcal{A}^{\prime}}\right)=\operatorname{dim}\left(\overline{\mathcal{M}}_{0, \mathcal{A}}\right)-k+1$.
} 
is non-negative on $B$. It is well-known that the divisor $\kappa+\psi=12 \lambda-\Delta+\psi$ is ample on $\overline{\mathcal{M}}_{g, n}[4,10]$. Hence for any $P \in \mathrm{NS}\left(\overline{\mathcal{M}}_{g, \mathcal{A}}\right) \otimes \mathbb{Q}$ satisfying $\|P\| \ll 1$, the divisor $f^{*}(P+\epsilon(\kappa+\psi))$ is an effective combination of an ample divisor and an $f$-exceptional divisor. It follows that $(P+\epsilon(\kappa+\psi)) \cdot B \geq 0$ and

$$
(A+P) \cdot B=(A(\epsilon)+\epsilon(\kappa+\psi)+P) \cdot B \geq 0 .
$$

Finally, suppose that two sections are coincident. Then $B$ lies in the image of some replacement morphism $\chi$. By Lemma 3.1 and the induction assumption, the divisor $A+P$ pulls back to a sum of an ample divisor and a $\psi$ class. Since the selfintersection of any section in a family of stable curves of positive genus is non-positive, we are done.

\section{Certain log canonical models of $\overline{\mathcal{M}}_{g, n}$}

We now apply the result of the previous section to establish ampleness of certain log canonical divisors on $\overline{\mathcal{M}}_{g, \mathcal{A}}$. To begin, by [12, Section 3.1.1] the canonical class of $\overline{\mathcal{M}}_{g, \mathcal{A}}$ is

$$
K_{\overline{\mathcal{M}}_{g, \mathcal{A}}}=13 \lambda-2 \Delta_{\text {nodal }}+\psi=\lambda+\kappa-\Delta_{\text {nodal }}+\psi .
$$

Thus, the ample divisor $A$ of Proposition 3.1 can be written as

$$
A=K_{\overline{\mathcal{M}}_{g, \mathcal{A}}}+\sum_{i<j}\left(a_{i}+a_{j}\right) \Delta_{i, j}+\Delta_{\text {nodal }}-\lambda .
$$

Since $\lambda$ is always nef, we see that $A+\lambda=K_{\overline{\mathcal{M}}_{g, \mathcal{A}}}+\sum_{i<j}\left(a_{i}+a_{j}\right) \Delta_{i, j}+\Delta_{\text {nodal }}$ is a natural ample $\log$ canonical divisor on $\overline{\mathcal{M}}_{g, \mathcal{A}}$. Using this, we can give an affirmative answer to [12, Problem 7.1]. The statement is cleanest for $g=0$, when all the spaces under consideration are smooth projective varieties.

Theorem 4.1. The coarse moduli space $\bar{M}_{g, \mathcal{A}}$ is a log canonical model of $\overline{\mathcal{M}}_{g, n}$. Namely,

$$
\begin{aligned}
\bar{M}_{g, \mathcal{A}}= & \operatorname{Proj} \bigoplus_{m \geq 0} \mathrm{H}^{0}\left(\overline{\mathcal{M}}_{g, n}, m\left(K_{\overline{\mathcal{M}}_{g, n}}+\sum_{\substack{i<j \\
a_{i}+a_{j} \leq 1}}\left(a_{i}+a_{j}\right) \Delta_{\{i, j\}}\right.\right. \\
& \left.\left.+\left(\Delta-\sum_{\substack{i<j \\
a_{i}+a_{j} \leq 1}} \Delta_{\{i, j\}}\right)\right)\right),
\end{aligned}
$$

where the sum is taken over sufficiently divisible $m$.

Proof. Consider the reduction morphism $f: \overline{\mathcal{M}}_{g, n} \rightarrow \overline{\mathcal{M}}_{g, \mathcal{A}}$. Observe that

$$
\begin{gathered}
f_{*}\left(K_{\overline{\mathcal{M}}_{g, n}}+\sum_{\substack{i<j \\
a_{i}+a_{j} \leq 1}}\left(a_{i}+a_{j}\right) \Delta_{\{i, j\}}+\left(\Delta-\sum_{\substack{i<j \\
a_{i}+a_{j} \leq 1}} \Delta_{\{i, j\}}\right)\right) \\
=K_{\overline{\mathcal{M}}_{g, \mathcal{A}}}+\sum_{i<j}\left(a_{i}+a_{j}\right) \Delta_{i, j}+\Delta_{\text {nodal }}=A+\lambda
\end{gathered}
$$

is ample by Proposition 3.1 and nefness of $\lambda$. Next, the discrepancy divisor

$$
\left(K_{\overline{\mathcal{M}}_{g, n}}+\sum_{\substack{i<j \\ a_{i}+a_{j} \leq 1}}\left(a_{i}+a_{j}\right) \Delta_{\{i, j\}}+\left(\Delta-\sum_{\substack{i<j \\ a_{i}+a_{j} \leq 1}} \Delta_{\{i, j\}}\right)\right)-f^{*}(A+\lambda)
$$


is an effective combination of exceptional divisors with all discrepancies in $[0, \infty)$. Indeed, as a routine computation with test families shows,

$$
\begin{aligned}
f^{*}(A+\lambda)= & \left(K_{\overline{\mathcal{M}}_{g, n}}+\sum_{\substack{i<j \\
a_{i}+a_{j} \leq 1}}\left(a_{i}+a_{j}\right) \Delta_{\{i, j\}}+\left(\Delta-\sum_{\substack{i<j \\
a_{i}+a_{j} \leq 1}} \Delta_{\{i, j\}}\right)\right) \\
+ & \sum_{\substack{S:|S| \geq 3 \\
\sum_{i \in S} a_{i} \leq 1}}(|S|-1)\left(1-\sum_{i \in S} a_{i}\right) \Delta_{S} .
\end{aligned}
$$

It follows that

$$
\begin{aligned}
\bar{M}_{g, \mathcal{A}}= & \operatorname{Proj} \bigoplus_{m \geq 0} \mathrm{H}^{0}\left(\overline{\mathcal{M}}_{g, \mathcal{A}}, m(A+\lambda)\right) \\
= & \operatorname{Proj} \bigoplus_{m \geq 0} \mathrm{H}^{0}\left(\overline{\mathcal{M}}_{g, n}, m\left(K_{\overline{\mathcal{M}}_{g, n}}+\sum_{\substack{i<j \\
a_{i}+a_{j} \leq 1}}\left(a_{i}+a_{j}\right) \Delta_{\{i, j\}}\right.\right. \\
& \left.\left.+\left(\Delta-\sum_{\substack{i<j \\
a_{i}+a_{j} \leq 1}} \Delta_{\{i, j\}}\right)\right)\right) .
\end{aligned}
$$

\section{Nef divisors on $\overline{\mathcal{M}}_{0, n}$}

5.1. The case of $g=0$. We keep the conventions of Sections 2 and 3.1. The canonical divisor of $\overline{\mathcal{M}}_{0, \mathcal{A}}$ has class $K_{\overline{\mathcal{M}}_{0, \mathcal{A}}}=\psi-2 \Delta_{\text {nodal }}$. We now restate Theorems 3.1 and 4.1 (together with Proposition 3.1) for $\overline{\mathcal{M}}_{0, n}$ :

Theorem 5.1. The divisor

$$
A\left(a_{1}, \ldots, a_{n}\right)=\psi+\sum_{i<j}\left(a_{i}+a_{j}\right) \Delta_{i j}-\Delta_{\text {nodal }},
$$

is ample on $\overline{\mathcal{M}}_{0, \mathcal{A}}$, and the divisors

$$
\begin{aligned}
& B\left(a_{1}, \ldots, a_{n}\right)=\sum_{i=1}^{n}\left(2 a_{i}-a_{i}^{2}\right) \psi_{i}+\sum_{i<j}\left(2 a_{i} a_{j}\right) \Delta_{i j}-\Delta_{\text {nodal }} \\
& C\left(a_{1}, \ldots, a_{n}\right)=\sum_{i=1}^{n}\left(1-a_{i}\right) \psi_{i}+\sum_{j \neq i}\left(a_{i}+a_{j}\right) \Delta_{i j}
\end{aligned}
$$

are nef on $\overline{\mathcal{M}}_{0, \mathcal{A}}$. 
Theorem 5.2. The Hassett's moduli space $\overline{\mathcal{M}}_{0, \mathcal{A}}$ is a log canonical model of $\overline{\mathcal{M}}_{0, n}$ :

$$
\begin{aligned}
\overline{\mathcal{M}}_{0, \mathcal{A}}= & \operatorname{Proj} \bigoplus_{m \geq 0} \mathrm{H}^{0}\left(\overline{\mathcal{M}}_{0, n}, m\left(K_{\overline{\mathcal{M}}_{0, n}}+\sum_{\substack{i<j \\
a_{i}+a_{j} \leq 1}}\left(a_{i}+a_{j}\right) \Delta_{\{i, j\}}\right.\right. \\
& \left.\left.+\left(\Delta-\sum_{\substack{i<j \\
a_{i}+a_{j} \leq 1}} \Delta_{\{i, j\}}\right)\right)\right),
\end{aligned}
$$

where the sum is taken over sufficiently divisible $m$.

5.2. $\mathrm{SL}_{2}$ quotients of $\left(\mathbb{P}^{1}\right)^{n}$. In [1], Alexeev and Swinarski introduced a subcone, called the GIT cone, of the nef cone of $\overline{\mathcal{M}}_{0, n}$. The GIT cone is generated by pullbacks of natural polarizations on GIT quotients $\left(\mathbb{P}^{1}\right)^{n} / /{ }_{x} \mathrm{SL}_{2}$. Here, we show that the GIT cone is contained ${ }^{3}$ in the closure of the cone generated by divisors of Theorem 5.1. The idea of proof is simple: We observe that the GIT polarization on $\left(\mathbb{P}^{1}\right)^{n} / / \vec{x}_{\vec{x}} \mathrm{SL}_{2}$ is proportional to the divisor class $A\left(x_{1}, \ldots, x_{n}\right)$ of Theorem 5.1.

In what follows, we regard $\left(\mathbb{P}^{1}\right)^{n} / / \vec{x} \mathrm{SL}_{2}$ as a good moduli space [2] of an Artin moduli stack $\left[\left(\mathbb{P}^{1}\right)^{n, \mathrm{ss}} / \mathrm{SL}_{2}\right]$, where the semistable locus is with respect to the linearization given by $\vec{x}$. When the linearization is typical, i.e., there are no strictly semistable points - no $x_{i}$ 's add up to 1 , the stack is Deligne-Mumford. In turn, we can regard $\left[\left(\mathbb{P}^{1}\right)^{n, \mathrm{ss}} / \mathrm{SL}_{2}\right]$ as the moduli stack of weighted $n$-pointed rational curves, where weights are given by $\vec{x}$. When the linearization is typical, we have $\left[\left(\mathbb{P}^{1}\right)^{n, \mathrm{ss}} / \mathrm{SL}_{2}\right]=\overline{\mathcal{M}}_{0, \mathcal{A}}$, where $a_{i}=x_{i}+\epsilon$ for $0<\epsilon \ll 1$. With these conventions, all tautological divisors introduced in Section 3.1 are still defined on $\left[\left(\mathbb{P}^{1}\right)^{n, \mathrm{ss}} / \mathrm{SL}_{2}\right]$ (but not all of them descend to $\left(\mathbb{P}^{1}\right)^{n} / /{ }_{\vec{x}} \mathrm{SL}_{2}$ when the linearization is atypical).

Proposition 5.1. Let $\vec{x}=\left(x_{1}, \ldots, x_{n}\right)$ be such that $x_{1}+\cdots+x_{n}=2$. Then the natural GIT polarization on $\left(\mathbb{P}^{1}\right)^{n} / / \mathrm{SL}_{2}$ is proportional to $A\left(x_{1}, \ldots, x_{n}\right)$.

Proof. We treat $\left(\mathbb{P}^{1}\right)^{n}$ as the parameter space of $n$ ordered points on $\mathbb{P}^{1}$. Consider the universal family $\pi:\left(\mathbb{P}^{1}\right)^{n} \times \mathbb{P}^{1} \rightarrow\left(\mathbb{P}^{1}\right)^{n}$ with sections $\tau_{1}, \ldots, \tau_{n}:\left(\mathbb{P}^{1}\right)^{n} \rightarrow\left(\mathbb{P}^{1}\right)^{n} \times \mathbb{P}^{1}$. For $i=1, \ldots, n+1$, let $H_{i}=\operatorname{pr}_{i}^{*} \mathcal{O}_{\mathbb{P}^{1}}(1)$ on $\left(\mathbb{P}^{1}\right)^{n} \times \mathbb{P}^{1}$. Then $\tau_{i}=H_{i}+H_{n+1}$, for $i=1, \ldots, n$, and the relative dualizing sheaf of the universal family is $\omega=-2 H_{n+1}$. We compute

$$
\left(\omega+\sum_{i=1}^{n} x_{i} \tau_{i}\right)\left(\omega+\sum_{i=1}^{n} \tau_{i}\right)=\left(\sum_{i=1}^{n} x_{i} H_{i}\right)\left((n-2) H_{n+1}+\sum_{i=1}^{n} H_{i}\right) .
$$

Pushing forward via $\pi$, we obtain that on $\left(\mathbb{P}^{1}\right)^{n}$

$$
\pi_{*}\left(\omega+\sum_{i=1}^{n} x_{i} \tau_{i}\right)\left(\omega+\sum_{i=1}^{n} \tau_{i}\right)=(n-2) \sum_{i=1}^{n} x_{i} H_{i} .
$$

By definition, some multiple of $\sum_{i=1}^{n} x_{i} H_{i}$ descends to the GIT polarization on $\left(\mathbb{P}^{1}\right)^{n} / /{ }_{x} \mathrm{SL}_{2}$. Hence, the divisor class $\pi_{*}\left(\omega+\sum_{i=1}^{n} x_{i} \tau_{i}\right)\left(\omega+\sum_{i=1}^{n} \tau_{i}\right)$ descends to a multiple of the GIT polarization. On the other hand, since $\omega$ and $\tau_{i}$ are defined

\footnotetext{
${ }^{3}$ We do not preclude a possibility that two cones coincide.
} 
functorially, the divisor class $\pi_{*}\left(\omega+\sum_{i=1}^{n} x_{i} \tau_{i}\right)\left(\omega+\sum_{i=1}^{n} \tau_{i}\right)$ descends to $A\left(x_{1}, \ldots, x_{n}\right)$ on $\left(\mathbb{P}^{1}\right)^{n} / / \vec{x} \mathrm{SL}_{2}$. The statement follows.

For completeness, we record what divisor classes on $\left(\mathbb{P}^{1}\right)^{n}$ descend to tautological divisor classes $\psi_{i}, \Delta_{i j}$ and $\Delta_{\text {nodal }}$ on $\left(\mathbb{P}^{1}\right)^{n} / /{ }_{\vec{x}} \mathrm{SL}_{2}$ when the linearization is typical. On $\left(\mathbb{P}^{1}\right)^{n}$ :

$$
\pi_{*}\left(-\tau_{i}^{2}\right)=\pi_{*}\left(-\left(H_{i}+H_{n+1}\right)^{2}\right)=-2 H_{i} .
$$

It follows that $-2 H_{i}$ descends to $\psi_{i}$. Further, since $\left(\tau_{i}-\tau_{j}\right)^{2}=0$ on $\left(\mathbb{P}^{1}\right)^{n} \times \mathbb{P}^{1}$, we conclude that

$$
\Delta_{i j}=-\frac{1}{2}\left(\psi_{i}+\psi_{j}\right)
$$

Finally, $\Delta_{\text {nodal }}=0$ because all curves parameterized by $\left(\mathbb{P}^{1}\right)^{n} / / \vec{x} \mathrm{SL}_{2}$ are irreducible.

We conclude that the GIT polarization on $\left(\mathbb{P}^{1}\right)^{n} / / \vec{x} \mathrm{SL}_{2}$ is written in terms of tautological divisor classes as

$$
A\left(x_{1}, \ldots, x_{n}\right)=\sum_{i=1}^{n} \psi_{i}+\sum_{i<j}\left(x_{i}+x_{j}\right) \Delta_{i j}-\Delta_{\text {nodal }}=-\frac{(n-2)}{2} \sum_{i=1}^{n} x_{i} \psi_{i} .
$$

\section{Acknowledgments}

We are grateful to Brendan Hassett for posing a question from which this paper originated and for useful comments regarding this work. We would like to thank David Smyth for helpful discussions and remarks on an early version of this paper. We thank Han-Bom Moon for pointing out an error in an old version of Lemma 3.1.

\section{References}

[1] V. Alexeev and D. Swinarski, Nef divisors on $\bar{M}_{0, n}$ from GIT (2008), arXiv:0810.1677 [math.AG].

[2] J. Alper, Good moduli spaces for Artin stacks (2008), arXiv:0810.1677 [math.AG].

[3] E. Arbarello and M. Cornalba, Calculating cohomology groups of moduli spaces of curves via algebraic geometry, Publ. Math. Inst. Hautes Études Sci. (88) (1998), 97-127.

[4] M. Cornalba and J. Harris, Divisor classes associated to families of stable varieties, with applications to the moduli space of curves, Ann. Sci. École Norm. Sup. (4) 21(3) (1988), 455-475.

[5] A. J. de Jong, Smoothness, semi-stability and alterations, Publ. Math. Inst. Hautes Études Sci. (83) (1996), 51-93.

[6] G. Farkas and A. Gibney, The Mori cones of moduli spaces of pointed curves of small genus, Trans. Amer. Math. Soc. 355(3) (2003), 1183-1199 (electronic).

[7] M. Fedorchuk and D. I. Smyth, Ample divisors on moduli spaces of pointed rational curves, J. Algebraic Geom. 20(4) (2011), 599-629.

[8] R. Friedman, Algebraic surfaces and holomorphic vector bundles, Universitext, Springer-Verlag, New York (1998).

[9] A. Gibney, Numerical criteria for divisors on $\bar{M}_{g}$ to be ample, Compos. Math. 145(5) (2009), $1227-1248$.

[10] A. Gibney, S. Keel and I. Morrison, Towards the ample cone of $\bar{M}_{g, n}$, J. Amer. Math. Soc. 15(2) (2002), 273-294 (electronic).

[11] J. Harris and I. Morrison, Moduli of curves, in "Graduate texts in mathematics", 187, SpringerVerlag, New York, 1998.

[12] B. Hassett, Moduli spaces of weighted pointed stable curves, Adv. Math. 173(2) (2003), 316-352.

[13] Y. Hu and S. Keel, Mori dream spaces and GIT, Michigan Math. J. 48 (2000), 331-348. Dedicated to William Fulton on the occasion of his 60th birthday. 
[14] S. Keel, Basepoint freeness for nef and big line bundles in positive characteristic, Ann. Math. (2) 149(1) (1999), 253-286.

[15] S. Keel and J. McKernan, Contractible extremal rays on $\bar{M}_{0, n}$ (1996), arXiv:9607.009.

[16] S. L. Kleiman, Toward a numerical theory of ampleness, Ann. Math. (2) 84 (1966), $293-344$.

[17] J. Kollár, Projectivity of complete moduli, J. Differential Geom. 32(1) (1990), 235-268.

[18] P. Larsen, Fulton's conjecture for $\bar{M}_{0,7}$ (2009), arXiv:0912.3104 [math.AG].

[19] M. Simpson, On Log canonical models of the moduli space of stable pointed genus zero curves, $\mathrm{PhD}$ thesis, Rice University, 2008.

Department of Mathematics, Columbia University, 2990 Broadway, New York, Ny 10027, USA

E-mail address: mfedorch@math.columbia.edu 
